\title{
Systematic review and meta-analysis of factors that help or hinder treatment decision-making capacity in psychosis
}

Amanda Larkin and Paul Hutton

\section{Background}

The evidence on factors that may influence treatment decisional capacity ('capacity') in psychosis has yet to be comprehensively synthesised, which limits the development of effective strategies to improve or support it.

\section{Aims \\ To determine the direction, magnitude and reliability of the relationship between capacity in psychosis and a range of clinical, demographic and treatment-related factors, thus providing a thorough synthesis of current knowledge.}

\section{Method}

We conducted a systematic review, meta-analytical and narrative synthesis of factors that help or hinder treatment decision-making capacity in psychosis, assessing the direction, magnitude, significance and reliability of reported associations.

\section{Results}

We identified 23 relevant studies $(n=1823)$. Psychotic

symptoms had small, moderate and strong associations with appreciation, understanding and reasoning respectively. Both verbal cognitive functioning and duration of education had small to moderate correlations with understanding and reasoning. Better capacity was also associated with better insight, better metacognitive ability, higher anxiety and lower perceived coercion. No linear relationship with depression was observed. Interventions linked to improved capacity over time were in-patient care, information simplification, shared decision-making and metacognitive training.

\section{Conclusions}

Although much is known about the role of symptoms and other clinical variables, effective and acceptable psychological interventions to support capacity in this group are lacking

\section{Declaration of interest}

None.

\section{Copyright and usage}

(c) The Royal College of Psychiatrists 2017.
Decisional capacity is a term that has its origins in legislation, but has considerable implications for clinical practice. Definitions vary but research in this area has centred on a model published by Paul Appelbaum and Thomas Grisso in 1995, who conceptualised capacity in terms of four abilities: ability to communicate a choice, ability to understand relevant information, ability to appreciate relevant information and ability to manipulate information rationally. ${ }^{1}$ In healthcare, treatment decision-making capacity - hereinafter referred to as 'capacity' - is closely related to agency (the capacity of a person, or 'agent', to take intentional action), autonomy and the exercise of self-governance, concepts that are fundamental to human dignity and rights. ${ }^{2}$ For example, Article 12 of the United Nations Convention on the Rights of Persons with Disabilities recognises the right to be recognised as a person before the law, and the subsequent right to have one's decisions legally recognised. Autonomy and empowerment are thought to be essential components of patient-defined recovery from psychosis, ${ }^{3,4}$ and mental health legislation frequently requires clinicians to empower patients to make decisions and to make an assumption of capacity until proved otherwise, e.g. the Adults with Incapacity (Scotland) Act (2000) and the Mental Capacity Act 2005. However, there is also a concern that if patients who lack capacity to make specific decisions are allowed to make these decisions, then these may not reflect their true wishes, with the consequence being a poor outcome and inadequate protection of the patient. ${ }^{5}$ Capacity has understandably been called the 'gatekeeper for autonomy' ${ }^{6}$ Lepping et al found that the average percentage of patients with impaired capacity on psychiatric wards is $45 \% .^{7}$ Despite the frequency with which psychiatrists are asked to make such judgements, almost half of them view the evidence base in this area as weak. ${ }^{9}$
Nonetheless, the field of decision-making capacity research has grown in recent years. This has been spurred on by changes in legislation, but also by a change in the culture in which healthcare decisions are made. People using mental health services are showing a greater desire to be included in decisions about their treatment, ${ }^{9}$ and there has been an increasing emphasis on ensuring not only that patients give informed consent to treatment, but also that they are actively involved in the decision-making process. ${ }^{10}$ The most common model for such involvement is called 'shared decision-making', but there is evidence that people with psychosis do not typically experience this. ${ }^{11}$ Since impaired capacity is a major barrier to psychiatrists implementing shared decision-making with people with psychosis, ${ }^{12}$ improving our understanding of factors that cause or maintain this impairment may help to change this. Moreover, British Medical Association guidance on assessing and managing capacity advises that it is the duty of the assessing clinician to enhance capacity where it is possible to do so. ${ }^{13}$ In the context of psychiatric and mental health conditions, this is often achieved through treatment of the condition itself; however, there has been little research on the effectiveness of current treatments for enhancing decision-making capacity. Although some studies have examined whether specific psychological and educational interventions can enhance capacity, ${ }^{14,15}$ the overall evidence is surprisingly limited.

Previous reviews have examined the prevalence of incapacity in psychiatric patients, ${ }^{16}$ the reliability and validity of measurement tools, ${ }^{17,18}$ the degree of impairment in decisional capacity in schizophrenia, ${ }^{19}$ the role of poor insight, ${ }^{20}$ and the role of specific neuropsychological deficits. ${ }^{21}$ Although one older review examined the correlates of capacity in psychiatric populations 
generally, ${ }^{16}$ no review has yet looked at the factors associated with capacity in psychosis specifically. Identifying these factors might help us develop a clinically useful theoretical model, which in turn would aid the development of effective interventions to support capacity. Thus, the primary objectives of this systematic review were to identify which clinical, demographic and treatmentrelated variables are associated with treatment decision-making capacity in psychosis and, where a sufficient number of comparable studies exist, to use meta-analysis to produce pooled estimates of the magnitude and reliability of any relationship.

\section{Method}

To minimise the risk of selective reporting bias and maximise transparency, a protocol for the systematic review was registered in advance with the International Prospective Register of Systematic Reviews (PROSPERO; registration number CRD42015025568). The protocol was updated to include a quantitative synthesis of effect sizes using meta-analytic procedures where three or more studies provided usable data, and incorporation of the Grades of Recommendation, Assessment, Development and Evaluation (GRADE) approach to assess outcome quality (online supplement DS1). ${ }^{22}$

\section{Inclusion and exclusion criteria}

Studies were included if they were published in English before October 2015, included a reliable and valid assessment of capacity in adults diagnosed with a non-affective psychotic disorder and provided data on the association between capacity and at least one other clinical or demographic variable (online supplement DS2). Assessment of capacity was accepted as valid if participants had been asked to make a real or hypothetical decision about a healthcare or treatment decision, and if a valid and reliable tool was used to measure at least one of the four accepted domains of decisional capacity described above. ${ }^{1}$ Studies reporting usable cross-sectional or longitudinal data were eligible for inclusion regardless of overall study design or purpose. Studies were excluded where the proportion of participants with non-affective psychosis was less than $50 \%$. Since we were specifically investigating correlates of treatment decision-making capacity, and because capacity is a decision-specific concept, we excluded studies where only capacity to consent to participate in research or legal proceedings was examined.

\section{Search strategy}

A search using the terms (Schizo* OR Psychosis) AND (Capacity OR Decision making OR Consent) AND (Treatment OR Health care) was conducted in the databases EMBASE, EMBASE Classic, Medline and PsycINFO from 1947 to October 2015. One researcher (A.L.) conducted the search (with support and training from a qualified librarian), and another (P.H.) provided supervision and consultation. Previous reviews and included studies were hand-searched for additional studies, and authors were contacted for any further unpublished studies.

\section{Study selection}

The titles and abstracts of studies identified by the search were screened to eliminate obviously ineligible studies such as studies of unrelated conditions, or other reviews. The full-text reports for any remaining studies were then examined to determine eligibility against the inclusion and exclusion criteria (online supplement DS3).

\section{Quality assessment}

In line with previous systematic reviews, ${ }^{23,24}$ the assessment of observational study quality was conducted using an adapted version of the Agency for Healthcare Research and Quality (AHRQ) assessment tool. ${ }^{25}$ The adequacy of the methods used to select the cohort, the sample size, the methods used to assess outcomes, the degree of missing data and the appropriateness of the analytic methods used were all assessed as 'yes', 'no', 'partial' or 'can't tell' (online supplement DS4). Randomised controlled trials were assessed using the well-established Cochrane Collaboration risk of bias tool, which assesses risk of selection, performance, detection, attrition and reporting biases. ${ }^{26}$ An adapted version of GRADE was used to assess the quality of the effect size estimates, whether derived from single studies or groups of studies. Specific criteria for assessing outcome quality within the GRADE approach are outlined in online supplement DS5.

\section{Statistical analysis}

Meta-analysis was conducted when at least three studies reported usable data on the relationship between a particular variable and treatment decision-making capacity. These were conducted using MetaXL software. Correlations were transformed into Fisher's $Z$, and a random effects model using the DerSimonian \& Laird method was used to compute an overall effect size, together with 95\% confidence intervals. ${ }^{27}$ This approach allows for true heterogeneity in effect size magnitude (due to differences in measurement, sample, etc.) to be distinguished from sampling error. ${ }^{28}$ Fisher's $Z$ estimates were then back-transformed to Pearson's $r$ to allow interpretation according to Cohen's 1988 conventions (0.1 small, 0.3 moderate, 0.5 large). ${ }^{29}$

\section{Results}

Of the 2057 papers initially identified, 1994 were excluded after inspection of title or abstract (Fig. 1). Full-text publications were

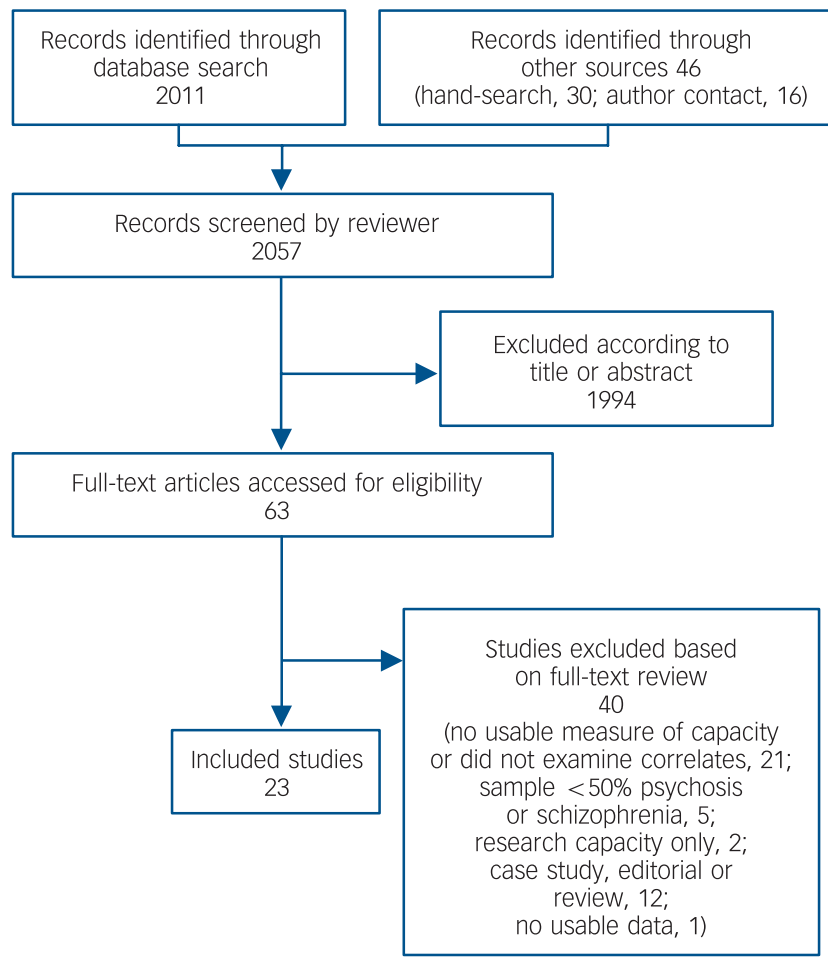

Fig. 1 Study selection 
sought for the remaining 63 papers. Of these, 40 were excluded: 21 did not include a measure of capacity or did not examine or report correlates, 12 were case descriptions, editorials or reviews, 4 examined a different population and 2 examined research decision-making capacity. A full list of excluded studies with reasons for exclusion is provided in online supplement DS3. A total of 23 studies were included for review (Table 1). These provided data on the relationship between capacity and symptoms $(k=12)$, insight $(k=4)$, affect $(k=3)$, cognitive performance $(k=6)$, executive functioning $(k=2)$, duration of illness $(k=2)$, education $(k=5)$, metacognition $(k=1)$ and various interventions $(k=10)$. 'Metacognition' refers to the implicit and explicit awareness, knowledge, beliefs and understanding we have about our cognitive systems and processes.

\section{Quality assessment}

Overall GRADE ratings for each outcome are presented in the right-hand columns of Tables 2-4. Quality ratings using the AHRQ measure of observational and uncontrolled intervention studies are shown in online Tables DS1 and DS2, and online Table DS3 provides Cochrane risk of bias ratings for randomised controlled trials. The studies generally performed well on the AHRQ and Cochrane risk of bias assessments. Methods used to assess key outcomes were generally reliable and valid, cohorts were as a rule well described and characterised, and most of the studies selected their participants in a relatively unbiased way (although convenience samples were widely used). The evidence was weakened by a general failure to provide prespecified power calculations. Although only a minority of studies $(k=5)$ had masked rater assessment of the relevant outcomes, we made a post hoc decision to exclude this from the quality assessment. Intervention studies often did not include a follow-up assessment. Funnel plots did not detect evidence of publication bias for the majority of the outcomes, but there were generally too few studies to assess this properly. ${ }^{30}$

\section{Meta-analysis}

\section{Psychotic symptoms}

As shown in Fig. 2, pooled data from nine studies $(n=610)$ suggested there was a moderate to large negative association between total psychotic symptom severity, as assessed by total Brief Psychiatric Rating Scale (BPRS) or Positive and Negative Syndrome Scale (PANSS) scores, and the capacity of participants to understand information relevant to treatment decisions ( $r=-0.45,95 \%$ CI -0.55 to $-0.34 ; I^{2}=60 \%$, moderate quality evidence). All studies reported a negative correlation between symptom severity and understanding, although one reported a considerably smaller effect size. ${ }^{31}$ Removing this led to a slightly larger correlation and lower heterogeneity $(r=-0.49,95 \% \mathrm{CI}$ -0.39 to $\left.-0.56 ; I^{2}=46 \%\right)$. Data from six studies $(n=453)$ suggested there was a small correlation between overall symptoms and the ability of participants to appreciate information relevant to a treatment decision $(r=-0.23,95 \%$ CI -0.14 to -0.32 ; $I^{2}=0 \%$, moderate quality evidence). According to data from seven studies $(n=528)$ there was a moderate correlation between total symptoms and the ability of participants to reason in relation to treatment decision-making $(r=-0.31,95 \% \mathrm{CI}-0.48$ to $-0.12 ; I^{2}=80 \%$ ), but the quality of the evidence was judged to be low because of risk of bias and high heterogeneity. This high heterogeneity appeared to be attributable to the large correlation reported by a study of a forensic in-patient sample; ${ }^{32}$ removing this study removed the heterogeneity and also lowered the effect size $\left(r=-0.24,95 \% \mathrm{CI}-0.33\right.$ to $\left.-0.14 ; I^{2}=0 \%\right)$.

\section{Depression}

There was no evidence that depression was associated with the ability of participants to understand information about their treatment (Fig. 3; $k=3, n=146 ; r=-0.04,95 \%$ CI -0.20 to $0.13, I^{2}=0 \%$; moderate quality evidence).

\section{Cognitive and intellectual performance}

Moderate to large associations were observed (Fig. 4) between verbal cognitive functioning (assessed using subtests from the Wechsler Adult Intelligence Scale) and the ability of participants to understand information relating to treatment decision-making $\left(k=4, n=203 ; r=0.42,95 \%\right.$ CI 0.20 to $0.60 ; I^{2}=60 \%$; low quality evidence) and to use reasoning ( $k=3, n=177 ; r=0.39,95 \% \mathrm{CI}$ 0.26 to $0.51 ; I^{2}=0 \%$; low quality evidence).

\section{Education}

Moderate-quality evidence suggested a large association between years spent in education and the ability of participants to understand information relating to treatment decisions (Fig. 5; $k=3, n=201 ; r=0.46,95 \%$ CI 0.36 to $\left.0.56 ; I^{2}=0 \%\right)$. The association between years of education and participants' reasoning ability was small to moderate ( $k=3, n=201 ; r=0.26,95 \%$ CI 0.12 to $0.38 ; I^{2}=0 \%$; moderate quality evidence).

\section{Outcomes from individual studies}

A full description of the results of individual studies is provided in online supplement DS6.

\section{Executive functioning}

One small study reported non-significant moderate correlations between domains of capacity and aspects of executive functioning, ${ }^{33}$ whereas another reported large reductions in capacity in those with poor executive functioning. ${ }^{34}$

Insight

Five studies assessed the relationship between capacity and different aspects of insight, ${ }^{35-39}$ and generally found large reductions in capacity in those with poor insight. One of these studies used the Beck Cognitive Insight Scale, ${ }^{39}$ and found much smaller and generally non-significant associations between capacity and self-certainty and self-reflectiveness, with the exception of reasoning and appreciation, which both had moderate positive correlations with self-reflectiveness (reasoning: $r=0.43,95 \%$ CI 0.20 to 0.62 ; appreciation: $r=0.33,95 \% 0.08$ to 0.54 ;). Overall we judged the evidence on insight to be of moderate quality and consistent with the view that insight is associated with improved capacity, in particular reasoning ability.

\section{Duration of illness}

Two studies provided low-quality data on the relationship between duration of illness and capacity. ${ }^{39,40}$ One did not find a relationship, ${ }^{39}$ whereas the other reported a small yet significant relationship with understanding $(r=-0.24,95 \% \mathrm{CI}-0.02$ to -0.44$) .{ }^{40}$

\section{Metacognitive ability}

One very small study found metacognitive ability was significantly associated with the understanding domain of capacity $(r=0.60$, $95 \%$ CI 0.23 to 0.82$).^{33}$ 
Table 1 Characteristics of included studies

\begin{tabular}{|c|c|c|c|c|c|c|c|c|}
\hline \multirow[b]{2}{*}{ Study } & \multicolumn{2}{|c|}{ Participants } & \multirow[b]{2}{*}{ Country } & \multirow[b]{2}{*}{$\begin{array}{l}\text { Measure of } \\
\text { capacity }\end{array}$} & \multirow[b]{2}{*}{$\begin{array}{l}\text { Variables measured } \\
\text { (name of measure) }\end{array}$} & \multicolumn{3}{|c|}{ Baseline demographics } \\
\hline & $\begin{array}{c}\text { Total } \\
n\end{array}$ & $\begin{array}{l}\text { Proportion with } \\
\text { psychosis, \% }\end{array}$ & & & & $\begin{array}{l}\text { Age, years: mean } \\
\text { (s.d. or range) }\end{array}$ & $\begin{array}{l}\text { Proportion } \\
\text { female, \% }\end{array}$ & $\begin{array}{l}\text { Treatment } \\
\text { setting }\end{array}$ \\
\hline Cairns et al $(2005)^{35}$ & 112 & 55 & England & MacCAT-T & $\begin{array}{c}\text { Psychotic symptoms (BPRS) } \\
\text { Insight (SAl-E) } \\
\text { Cognition (MMSE) } \\
\text { Coercion (BPCS) }\end{array}$ & $37.2(11.8)$ & 37 & In-patient \\
\hline Capdevielle et al (2009) & 60 & 100 & France & MacCAT-T & $\begin{array}{c}\text { Psychotic symptoms (PANSS) } \\
\text { Insight (SUMD) } \\
\text { Depression (BDI-2) } \\
\text { Anxiety (STAl) }\end{array}$ & $36.3(10.9)$ & 28 & Out-patient \\
\hline Di \& Chen $(2013)^{70}$ & 192 & 100 & China & SSICA & $\begin{array}{l}\text { Psychotic symptoms (BPRS) } \\
\text { Years of education }\end{array}$ & $30.3(15.2)$ & 19 & In-patient \\
\hline Dornan et al $(2015)^{48}$ & 37 & 89 & Ireland & MacCAT-T & $\begin{array}{l}\text { Psychotic symptoms (PANSS) } \\
\text { Functioning (GAF) }\end{array}$ & $\begin{array}{l}32.3 \\
(19.8-56.4)\end{array}$ & 8 & $\begin{array}{l}\text { In-patient } \\
\text { (forensic) }\end{array}$ \\
\hline Elbogen et al $(2007)^{38}$ & 469 & 59 & USA & DCAT-PAD & $\begin{array}{l}\text { Psychotic symptoms (BPRS) } \\
\text { Functioning (GAF) } \\
\text { Insight (ITAQ) } \\
\text { Cognition (AMNART; WAIS-II; } \\
\text { COWAT; HVLT) }\end{array}$ & $42(10.7)$ & 60 & Out-patient \\
\hline $\begin{array}{l}\text { Grisso \& Applebaum } \\
(1991)^{71}\end{array}$ & 26 & 100 & USA & MUD & $\begin{array}{c}\text { Psychotic symptoms (BPRS) } \\
\text { Depression (BDI) } \\
\text { Cognition (WAIS-R) }\end{array}$ & 36.8 (NS) & 31 & In-patient \\
\hline $\begin{array}{l}\text { Grisso \& Applebaum } \\
(1995)^{72}\end{array}$ & 75 & 100 & USA & $\begin{array}{l}\text { UTD, POD, } \\
\text { TRAT }\end{array}$ & $\begin{array}{c}\text { Psychotic symptoms (BPRS) } \\
\text { Depression (BDI) } \\
\text { Cognition (WAIS-R) }\end{array}$ & $35.4(7.4)$ & 48 & In-patient \\
\hline Grisso et al (1997) $)^{31}$ & 40 & 100 & USA & MacCAT-T & Psychotic symptoms (BPRS) & 39 (NS) & 20 & In-patient \\
\hline Hamann et al (2011) ${ }^{49}$ & 61 & 100 & Germany & Clinical & $\begin{array}{l}\text { Controlled trial; no correlational } \\
\text { data reported }\end{array}$ & $40.7(11.7)$ & 62 & In-patient \\
\hline Howe et al (2005) & 110 & 81 & Australia & MacCAT-T & Psychotic symptoms (PANSS) & $37.2(12.3)$ & 51 & In-patient \\
\hline Kennedy et al (2009) ${ }^{45}$ & 88 & 74 & Ireland & MaCAT-T & $\begin{array}{l}\text { Uncontrolled trial; no other } \\
\text { correlational data reported }\end{array}$ & NS & 9 & $\begin{array}{l}\text { In-patient } \\
\text { (forensic) }\end{array}$ \\
\hline Koren et al $(2005)^{33}$ & 21 & 100 & Israel & MacCAT-T & $\begin{array}{l}\text { Metacognition (WCST) } \\
\text { Cognition (WAIS-R) }\end{array}$ & $23.9(4.5)$ & 38 & In-patient \\
\hline Kleinman et al (1996) ${ }^{44}$ & 26 & 100 & Canada & $\begin{array}{l}\text { Knowledge } \\
\text { of medication }\end{array}$ & $\begin{array}{l}\text { Controlled trial; no correlational } \\
\text { data reported }\end{array}$ & NS & NS & In-patient \\
\hline Mandarelli et al (2012) ${ }^{34}$ & 45 & 56 & France & MacCAT-T & $\begin{array}{c}\text { Psychotic symptoms (BPRS) } \\
\text { Cognition (WCST) } \\
\text { Cognition (MMSE) }\end{array}$ & $41(13.1)$ & 55 & In-patient \\
\hline Munetz \& Roth $(1985)^{42}$ & 25 & 88 & USA & Questionnaire & $\begin{array}{l}\text { Uncontrolled trial; no other } \\
\text { correlational data reported }\end{array}$ & 48.6 (NS) & 66 & NS \\
\hline Naughton et al (2012) & 19 & 95 & Ireland & MacCAT-T & $\begin{array}{l}\text { Uncontrolled trial; no other } \\
\text { correlational data reported }\end{array}$ & $36.7(10.6)$ & 100 & $\begin{array}{l}\text { In-patient } \\
\text { (forensic) }\end{array}$ \\
\hline Owen et al $(2008)^{74}$ & 40 & 100 & England & MacCAT-T & $\begin{array}{l}\text { Psychotic symptoms (BPRS) } \\
\text { Cognition (WAIS-R) } \\
\text { Insight (SAl-E) }\end{array}$ & NS & NS & In-patient \\
\hline Palmer et al (2002) ${ }^{43}$ & 16 & 94 & USA & $\begin{array}{l}\text { MacCAT-T; } \\
\text { HCAT }\end{array}$ & $\begin{array}{c}\text { Psychotic symptoms (PANSS, } \\
\text { BPRS) } \\
\text { Cognition (DRS) }\end{array}$ & $54.6(7.2)$ & 44 & Out-patient \\
\hline Raffard et al (2013) ${ }^{39}$ & 60 & 100 & France & MacCAT-T & $\begin{array}{c}\text { Psychotic symptoms (PANSS) } \\
\text { Insight (BCIS) } \\
\text { Depression (BDI-2) } \\
\text { Anxiety (STAl) }\end{array}$ & $36.8(11.1)$ & 32 & Out-patient \\
\hline Rutledge et al (2008) $)^{32}$ & 102 & 88 & Ireland & MacCAT-T & $\begin{array}{l}\text { Psychotic symptoms (PANSS) } \\
\text { Functioning (GAF) }\end{array}$ & $38.1(16.2)$ & 9 & $\begin{array}{l}\text { In-patient } \\
\text { (forensic) }\end{array}$ \\
\hline Schacter et al (1994) ${ }^{75}$ & 59 & 100 & Canada & Questionnaire & Psychotic symptoms (BPRS) & 37 (NS) & 17 & Out-patient \\
\hline Wong et al $(2000)^{46}$ & 19 & 100 & England & Interview & Psychotic symptoms (BPRS) & $40.1(10.6)$ & 24 & Out-patient \\
\hline Wong et al $(2005)^{40}$ & 81 & 100 & Hong Kong & MacCAT-T & $\begin{array}{l}\text { Psychotic symptoms (PANSS) } \\
\text { Depression (MADRS) } \\
\text { Insight (DAI) } \\
\text { Cognition } \\
\text { (WAIS-R-HK; WCST; WMS; MCT) }\end{array}$ & $36.9(10.4)$ & 46 & In-patient \\
\hline 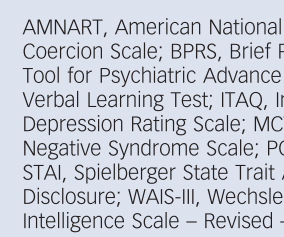 & $\begin{array}{l}\text { sht and } \\
\text { Monoton } \\
\text { Percept } \\
\text { ciety Inv } \\
\text { dult Inte } \\
\text { ong Kon }\end{array}$ & $\begin{array}{l}\text { BCIS, Beck Cognitive } \\
\text { ing Scale; CoWAT, CC } \\
\text { IS, Mattis Dementia R } \\
\text { atment Attitudes Que } \\
\text { Counting Test; MMSE, } \\
\text { is of Disorder; SAl-E, } \\
\text { ory; SUMD, scale to } \\
\text { ence Scale - 3rd edn } \\
\text { WCST, Wisconsin Car }\end{array}$ & $\begin{array}{l}\text { e Insight Scale; } \\
\text { Controlled Oral } \\
\text { Rating Scale; GA } \\
\text { lestionnaire; Ma } \\
\text { E, Mini Mental S } \\
\text {, Expanded Sche } \\
\text { Assess Unawar } \\
\text { n; WAIS-R, Wech } \\
\text { rd Sorting Task; }\end{array}$ & $\begin{array}{l}\text {; BDI, Beck Depres } \\
\text { Word Association } \\
\text { AaF, Global Assess } \\
\text { acCAT-T, MacArthn } \\
\text { State Examination; } \\
\text { nedule for the Asss } \\
\text { reness of Mental I } \\
\text { chsler Adult Intellig } \\
\text {; WMS, Wechsler I }\end{array}$ & $\begin{array}{l}\text { ession Inventory; BDI-2, Beck Depressio } \\
\text { TT Test; DAI, Drug Attitude Inventory; DC } \\
\text { sment of Functioning; HCAT, Hopkins C } \\
\text { hur Competence Assessment Tool-Trea } \\
\text { T; MUD, Measuring Understanding of D } \\
\text { sessment of Insight; ;SIICA, Semi-struct } \\
\text { I Disorder; TRAT, Thinking Rationally ab } \\
\text { igence Scale-Revised; WCST, Wisconsin } \\
\text { Memory Scale. }\end{array}$ & $\begin{array}{l}\text { ion Inventory - 2nd edn } \\
\text { CAT-PAD, Decisional C } \\
\text { Competency Assessme } \\
\text { atment; MADRS, Mont } \\
\text { Disclosure; NS, not stat } \\
\text { ctured Inventory for Co } \\
\text { bout Treatment; UTD, } \\
\text { in Card Sorting Test; W }\end{array}$ & $\begin{array}{l}\text { In; BPCS, Brief } \\
\text { Competence A } \\
\text { nent Test; HVL } \\
\text { tgomery \& Ast } \\
\text { ted; PANSS, P } \\
\text { ompetence As } \\
\text { Understanding } \\
\text { VAIS-R-HK, We }\end{array}$ & $\begin{array}{l}\text { "Perceived } \\
\text { Assessment } \\
\text { T, Hopkins } \\
\text { berg } \\
\text { ositive And } \\
\text { sessment; } \\
\text { g Treatment } \\
\text { chsler Adult }\end{array}$ \\
\hline
\end{tabular}




\begin{tabular}{|c|c|c|c|c|c|}
\hline $\begin{array}{l}\text { Outcome } \\
\text { (number of studies) }\end{array}$ & Included studies & $n$ & $\begin{array}{l}\text { Pooled Fisher's Z }(95 \% \mathrm{Cl}) \\
\text { Pooled } r(95 \% \mathrm{Cl})\end{array}$ & $\begin{array}{l}\text { Heterogeneity } \\
\left(I^{2} \text { for } Z\right), \%\end{array}$ & $\begin{array}{l}\text { Quality } \\
\text { (GRADE) }\end{array}$ \\
\hline $\begin{array}{l}\text { Relationship between total } \\
\text { symptom severity and } \\
\text { understanding ( } 9 \text { studies) }\end{array}$ & 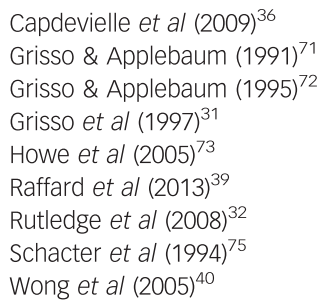 & 610 & $\begin{array}{l}Z=-0.49(-0.62 \text { to }-0.35) \\
r=-0.45(-0.55 \text { to }-0.33)\end{array}$ & 60 & $\begin{array}{c}\text { Moderate } \\
(-1 \text { risk of bias })\end{array}$ \\
\hline $\begin{array}{l}\text { Relationship between total } \\
\text { symptom severity and } \\
\text { appreciation ( } 6 \text { studies) }\end{array}$ & $\begin{array}{l}\text { Capdevielle et al (2009) } \\
\text { Grisso et al }(1997)^{31} \\
\text { Howe et al }(2005)^{73} \\
\text { Raffard et al }(2013)^{39} \\
\text { Rutledge et al }(2008)^{32} \\
\text { Wong et al }(2005)^{40}\end{array}$ & 453 & $\begin{aligned} Z & =-0.24(-0.33 \text { to }-0.14) \\
r & =-0.23(-0.14 \text { to }-0.32)\end{aligned}$ & 0 & $\begin{array}{c}\text { Moderate } \\
(-1 \text { risk of bias })\end{array}$ \\
\hline $\begin{array}{l}\text { Relationship between total } \\
\text { symptom severity and } \\
\text { reasoning ( } 7 \text { studies) }\end{array}$ & $\begin{array}{l}\text { Capdevielle et al (2009) } \\
\text { Grisso \& Applebaum }(1995)^{72} \\
\text { Grisso et al }(1997)^{31} \\
\text { Howe et al }(2005)^{73} \\
\text { Raffard et al }(2013)^{39} \\
\text { Rutledge et al }(2008)^{32} \\
\text { Wong et al }(2005)^{40}\end{array}$ & 528 & $\begin{array}{l}Z=-0.32(-0.52 \text { to }-0.12) \\
r=-0.31(-0.48 \text { to }-0.12)\end{array}$ & 80 & $\begin{array}{l}\text { Low } \\
(-1 \text { risk of bias, } \\
-1 \text { inconsistency })\end{array}$ \\
\hline $\begin{array}{l}\text { Relationship between } \\
\text { depression and understanding } \\
\text { (3 studies) }\end{array}$ & $\begin{array}{l}\text { Capdevielle et al (2009) })^{36} \\
\text { Grisso \& Applebaum }(1991)^{71} \\
\text { Raffard et al (2013) }\end{array}$ & 146 & $\begin{array}{l}Z=-0.04(-0.21 \text { to } 0.13) \\
r=-0.04(-0.20 \text { to } 0.13)\end{array}$ & 0 & $\begin{array}{c}\text { Moderate } \\
\text { (-1 imprecision) }\end{array}$ \\
\hline $\begin{array}{l}\text { Relationship between verbal } \\
\text { IQ and understanding } \\
\text { (4 studies) }\end{array}$ & $\begin{array}{l}\text { Grisso \& Applebaum (1991) } \\
\text { Grisso \& Applebaum }(1995)^{72} \\
\text { Koren et al }(2005)^{33} \\
\text { Wong et al (2005) }\end{array}$ & 203 & $\begin{array}{l}Z=0.45(0.20 \text { to } 0.69) \\
r=0.42(0.20 \text { to } 0.60)\end{array}$ & 60 & $\begin{array}{l}\text { Low } \\
(-1 \text { risk of bias, } \\
-1 \text { imprecision) }\end{array}$ \\
\hline $\begin{array}{l}\text { Relationship between verbal } \\
\text { IQ and reasoning } \\
\text { (3 studies) }\end{array}$ & $\begin{array}{l}\text { Grisso \& Applebaum }(1995)^{72} \\
\text { Koren et al }(2005)^{33} \\
\text { Wong et al }(2005)^{40}\end{array}$ & 177 & $\begin{array}{l}Z=0.42(0.27 \text { to } 0.57) \\
r=0.39(0.26 \text { to } 0.51)\end{array}$ & 0 & $\begin{array}{l}\text { Low }(-1 \text { risk of bias, } \\
-1 \text { imprecision })\end{array}$ \\
\hline $\begin{array}{l}\text { Relationship between } \\
\text { years of education and } \\
\text { understanding ( } 3 \text { studies) }\end{array}$ & $\begin{array}{l}\text { Capdevielle et al (2009) } \\
\text { Raffard et al }(2013)^{39} \\
\text { Wong et al }(2005)^{40}\end{array}$ & 201 & $\begin{array}{l}Z=0.49(0.35 \text { to } 0.63) \\
r=0.46(0.34 \text { to } 0.56)\end{array}$ & 0 & $\begin{array}{c}\text { Moderate } \\
(-1 \text { imprecision })\end{array}$ \\
\hline $\begin{array}{l}\text { Relationship between } \\
\text { years of education and } \\
\text { reasoning ( } 3 \text { studies) }\end{array}$ & $\begin{array}{l}\text { Capdevielle et al (2009) } \\
\text { Raffard et al }(2013)^{39} \\
\text { Wong et al }(2005)^{40}\end{array}$ & 201 & $\begin{array}{l}Z=0.26(0.12 \text { to } 0.40) \\
r=0.26(0.12 \text { to } 0.38)\end{array}$ & 0 & $\begin{array}{c}\text { Moderate } \\
\text { (-1 imprecision) }\end{array}$ \\
\hline
\end{tabular}

\section{Perceived coercion}

Moderate-quality evidence from one study suggested that participants without capacity reported higher levels of perceived coercion (Mann-Whitney $U=422.5, P<0.001$ ). ${ }^{35}$

Anxiety

Moderate-quality evidence from two studies suggested state and trait anxiety might be positively associated with aspects of capacity, i.e. greater anxiety was linked to greater treatment decisional capacity. ${ }^{36,39}$

\section{Interventions}

Of the ten intervention studies we identified, five assessed the effect of altering the presentation of information on capacity, two examined the effect of usual treatment, two examined the effect of shared decision-making and one examined the effect of metacognitive training, which is a form of psychological intervention designed to improve a person's awareness of cognitive biases and thinking styles that may be involved in psychotic symptoms. ${ }^{41}$

\section{Presentation of material}

Repetition of information, and discussion of presented information with others, were associated with significant large increases in capacity in two studies $(d=1.83,95 \%$ CI 0.48 to 3.18; $\left.\chi^{2}=12.05, P=0.002\right),{ }^{42,43}$ whereas a non-significant, small improvement was reported by a third $(d=0.27,95 \%$ CI -0.51 to 1.06$).^{44}$ However, Kennedy et al found that providing extra information to participants in a forensic setting was associated with a significant fall in capacity $(d=0.75,95 \%$ CI 0.30 to 1.20 ), with a statistically significant proportion of the sample becoming incapable of making a treatment choice following the presentation of extra information. ${ }^{45}$ Wong et al successively simplified the presentation of information and found that as the task was simplified, capacity improved significantly (Cochran's $Q=14.4$, d.f. $=3, P<0.01) .{ }^{46}$ Overall, the risk of bias across these studies suggested the evidence was of low quality.

\section{Usual treatment}

Owen et al found that $37 \%$ of patients regained capacity following a month of treatment in hospital. ${ }^{47}$ Dornan et al found that patients receiving treatment as usual, which included 25 hours per week of individual programmed activities as well as treatment with antipsychotic medications, improved on all domains of capacity: understanding $d=0.62 \quad(95 \%$ CI 0.15 to 1.09$)$; appreciation $d=0.39(95 \%$ CI -0.07 to 0.85$)$; reasoning $d=0.63$ (95\% CI 0.16 to 1.09$){ }^{48}$ These authors also found that patients treated with clozapine had significantly larger 


\begin{tabular}{|c|c|c|c|c|c|}
\hline $\begin{array}{l}\text { Correlate } \\
\text { (number of studies) }\end{array}$ & Studies included & $n$ & $\begin{array}{c}\text { Outcome measures } \\
\text { used }\end{array}$ & Key findings & $\begin{array}{l}\text { Quality } \\
\text { (GRADE) }\end{array}$ \\
\hline $\begin{array}{l}\text { Executive functioning } \\
\text { ( } 2 \text { studies) }\end{array}$ & $\begin{array}{l}\text { Koren et al }(2005)^{33} \\
\text { Mandarelli et al (2012) }\end{array}$ & 66 & WCST & $\begin{array}{c}\text { Some evidence of large correlations } \\
\text { in one study, but no clear evidence } \\
\text { in the other }\end{array}$ & $\begin{array}{l}\text { Very low } \\
(-1 \text { risk of bias, } \\
-1 \text { inconsistency, } \\
-1 \text { imprecision) }\end{array}$ \\
\hline Insight (5 studies) & $\begin{array}{l}\text { Cairns et al }(2005)^{35} \\
\text { Capdevielle et al (2009) } \\
\text { Owen et al }(2009)^{37} \\
\text { Raffard et al }(2013)^{39} \\
\text { Elbogen et al }(2007)^{38}\end{array}$ & 813 & SUMD, SAI-E, BCIS, ITAQ & $\begin{array}{l}\text { Insight strongly and significantly } \\
\text { associated with capacity, } \\
\text { and reasoning in particular }\end{array}$ & $\begin{array}{c}\text { Moderate } \\
(-1 \text { risk of bias, } \\
-1 \text { indirectness, } \\
+1 \text { large effects) }\end{array}$ \\
\hline $\begin{array}{l}\text { Duration of illness } \\
\text { ( } 2 \text { studies) }\end{array}$ & $\begin{array}{l}\text { Raffard et al }(2013)^{39} \\
\text { Wong et al }(2005)^{40}\end{array}$ & 141 & Years since diagnosis & $\begin{array}{l}\text { Some evidence of small correlation } \\
\text { in one study, but no clear evidence } \\
\text { in the other }\end{array}$ & $\begin{array}{l}\text { Low } \\
\text { ( }-1 \text { risk of bias, } \\
-1 \text { imprecision) }\end{array}$ \\
\hline $\begin{array}{l}\text { Metacognitive ability } \\
\text { (1 study) }\end{array}$ & Koren et al $(2005)^{33}$ & 21 & $\begin{array}{l}\text { Participant ratings of } \\
\text { confidence in the } \\
\text { correctness of the sort } \\
(0-100)\end{array}$ & $\begin{array}{l}\text { Metacognitive ability found to be } \\
\text { associated with capacity }\end{array}$ & $\begin{array}{c}\text { Moderate } \\
\text { (-2 imprecision, } \\
+1 \text { large effect) }\end{array}$ \\
\hline $\begin{array}{l}\text { Perceived coercion } \\
\text { (1 study) }\end{array}$ & Cairns et al $(2005)^{35}$ & 112 & BPCS & $\begin{array}{l}\text { Participants judged to have impaired } \\
\text { capacity were more likely to report high } \\
\text { perceived coercion }\end{array}$ & $\begin{array}{c}\text { Moderate } \\
(-1 \text { imprecision })\end{array}$ \\
\hline Anxiety (2 studies) & $\begin{array}{l}\text { Capdevielle et al (2009) } \\
\text { Raffard et al }(2013)^{39}\end{array}$ & 120 & STAl & $\begin{array}{l}\text { Both state and trait anxiety had small } \\
\text { to medium positive correlations with } \\
\text { appreciation and reasoning, but not } \\
\text { with understanding or communicating }\end{array}$ & $\begin{array}{c}\text { Moderate } \\
(-1 \text { imprecision })\end{array}$ \\
\hline
\end{tabular}

Insight; STAI, Spielberger State-Trait Anxiety Inventory; SUMD, Scale to Assess Unawareness of Mental Disorder; WCST, Wisconsin Card Sorting Test.

\begin{tabular}{|c|c|c|c|c|c|}
\hline Intervention & Studies included & $n$ & Outcome measure & Key finding & Quality (GRADE) \\
\hline $\begin{array}{l}\text { Altering presentation } \\
\text { of material ( } 5 \text { studies) }\end{array}$ & $\begin{array}{l}\text { Kennedy et al }(2009)^{45} \\
\text { Kleinman et al (1996) } \\
\text { Munetz \& Roth }(1985)^{42} \\
\text { Palmer et al }(2002)^{43} \\
\text { Wong et al }(2000)^{46}\end{array}$ & 176 & $\begin{array}{l}\text { Change in capacity } \\
\text { scores }\end{array}$ & $\begin{array}{l}\text { Altering presentation of material } \\
\text { associated with improved capacity }\end{array}$ & $\begin{array}{c}\text { Low } \\
(-2 \text { risk of bias })\end{array}$ \\
\hline $\begin{array}{l}\text { Treatment as usual } \\
\text { (antipsychotic medication) } \\
\text { (2 studies) }\end{array}$ & $\begin{array}{l}\text { Dornan et al }(2015)^{48} \\
\text { Owen et al }(2011)^{47}\end{array}$ & 237 & $\begin{array}{l}\text { Change in capacity } \\
\text { scores }\end{array}$ & $\begin{array}{l}\text { Treatment as usual (including } \\
\text { antipsychotics) associated with } \\
\text { improved capacity }\end{array}$ & $\begin{array}{c}\text { Moderate } \\
(-2 \text { risk of bias, } \\
+1 \text { large effect })\end{array}$ \\
\hline $\begin{array}{l}\text { Shared decision-making } \\
\text { ( } 2 \text { studies) }\end{array}$ & $\begin{array}{l}\text { Elbogen et al }(2007)^{38} \\
\text { Hamann et al }(2011)^{49}\end{array}$ & 442 & $\begin{array}{l}\text { Change in capacity } \\
\text { scores }\end{array}$ & $\begin{array}{l}\text { SDM improved capacity in one } \\
\text { trial, but not in the other }\end{array}$ & $\begin{array}{l}\text { Low } \\
(-1 \text { risk of bias, } \\
-1 \text { inconsistency) }\end{array}$ \\
\hline $\begin{array}{l}\text { Metacognitive training } \\
\text { (1 study) }\end{array}$ & Naughton et al (2012) & 19 & $\begin{array}{l}\text { Change in capacity } \\
\text { scores }\end{array}$ & $\begin{array}{l}\text { MCT associated with improved } \\
\text { capacity scores }\end{array}$ & $\begin{array}{l}\text { Low } \\
\text { (-1 risk of bias, } \\
-2 \text { imprecision, } \\
+1 \text { large effects) }\end{array}$ \\
\hline
\end{tabular}

improvements in appreciation than patients treated with other antipsychotic agents $(d=2.10,95 \%$ CI 1.15 to 3.05$)$, and smaller non-significant improvements were also observed for understanding ( $d=0.75,95 \% \mathrm{CI}-0.09$ to 1.59$)$ and reasoning ( $d=0.71,95 \%$ CI -0.13 to 1.55$)$. Overall, the evidence for the effect of usual treatment, including antipsychotic medication, was judged to be moderate in quality, with the risk of bias across the studies being mitigated by the large observed effects.

\section{Shared decision-making}

Two trials examined the effect of a shared decision-making intervention on capacity. However, these studies found conflicting results, meaning the overall estimate was low in quality. Elbogen et al found a significant effect of shared decision-making on reasoning $\left(F_{(1,355)}=4.30, P<0.05\right)$ but not on appreciation or understanding, ${ }^{38}$ whereas Hamann et al found a non-significant small negative effect on capacity $(d=-0.34,95 \% \mathrm{CI}-0.85$ to 0.16$) .{ }^{49}$

\section{Metacognitive training}

In a small uncontrolled study, Naughton et al found that patients who received group metacognitive training had significantly improved understanding ( $d=1.44,95 \%$ CI 0.42 to 2.45$)$ and reasoning ability $(d=1.21,95 \%$ CI 0.22 to 2.20$)$, but there was no evidence of improvement in appreciation $(d=0.19,95 \% \mathrm{CI}$ -0.72 to 1.10$).{ }^{15}$

\section{Discussion}

Our primary objective was to identify which clinical, demographic and intervention-related variables are associated with treatment decision-making capacity in psychosis, and to assess the direction, magnitude and reliability of any relationships. Taken together, our findings suggest that individuals with psychosis are at high risk of being judged to lack capacity if they have spent less time in education, if they disagree with their clinician that they are ill 
(a) Study

Capdevielle et al (2009) $)^{36}$ Raffard et al (2013) ${ }^{39}$ Rutledge et al (2008) ${ }^{32}$ Wong et al (2005) ${ }^{40}$ Grisso \& Applebaum (1995) 72 Grisso et al (1997) $)^{31}$ Grisso \& Applebaum (1991) Howe et al (2005) ${ }^{73}$ Schacter et al (1994) ${ }^{75}$

Overall $Q=20.14, P=0.01, I^{2}=60 \%$

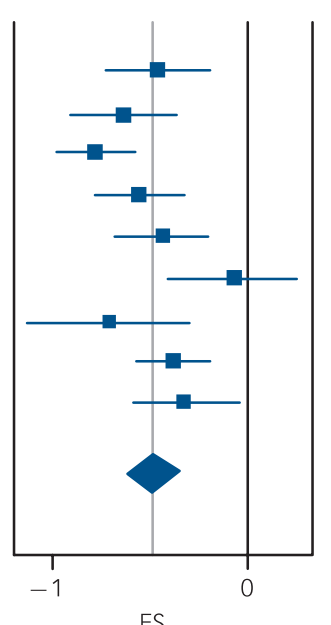

ES $(95 \% \mathrm{Cl}) \quad$ \% Weight

$-0.46(-0.72,-0.20) \quad 11.0$

$-0.63(-0.89,-0.37) \quad 11.0$

$-0.78(-0.97,-0.58) \quad 13.4$

$-0.55(-0.77,-0.33) \quad 12.4$

$-0.44(-0.67,-0.20) \quad 12.1$

$-0.07(-0.39,0.25) \quad 9.0$

$-0.71(-1.12,-0.30) \quad 6.8$

$-0.38(-0.57,-0.19) \quad 13.7$

$-0.32(-0.59,-0.05) \quad 10.7$

$-0.49(-0.62,-0.35) \quad 100.0$

(b) Study

Capdevielle et al (2009) $)^{36}$

Raffard et al (2013) $)^{39}$

Rutledge et al (2008) $)^{32}$

Wong et al (2005) $)^{40}$

Grisso et al (1997) ${ }^{31}$

Howe et al (2005)

Overall

$Q=2.58, P=0.77, I^{2}=0 \%$

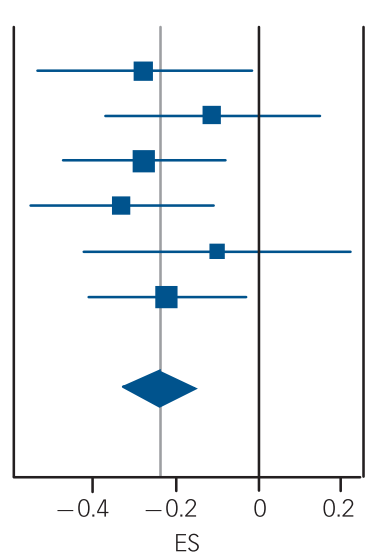

$\begin{array}{cc}\text { ES }(95 \% \mathrm{Cl}) & \text { \% Weight } \\ -0.28(-0.54,-0.02) & 13.1 \\ -0.11(-0.37,-0.15) & 13.1 \\ -0.28(-0.47,-0.08) & 22.8 \\ -0.33(-0.55,-0.11) & 17.9 \\ -0.10(-0.42,-0.22) & 9.5 \\ -0.22(-0.41,0.03) & 24.6 \\ -0.24(-0.33,-0.14) & 100.0\end{array}$

(c) Study

Capdevielle et al (2009) $)^{36}$ Raffard et al (2013) $)^{39}$ Rutledge et al (2008) ${ }^{32}$ Wong et al (2005) ${ }^{40}$ Grisso \& Applebaum (1995) ${ }^{72}$ Grisso et al (1997) ${ }^{31}$ Howe et al (2005) ${ }^{73}$

Overall

$Q=0.97, P=0.62, I^{2}=0 \%$

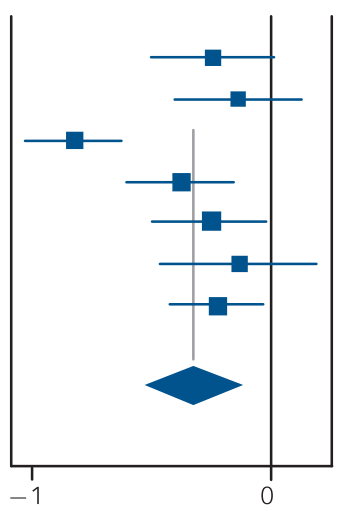

$$
\begin{array}{cc}
\text { ES }(95 \% \mathrm{Cl}) & \text { \% Weight } \\
-0.24(-0.50,-0.01) & 13.8 \\
-0.13(-0.39,-0.13) & 13.8 \\
-0.83(-1.03,-0.63) & 15.3 \\
-0.38(-0.60,-0.15) & 14.7 \\
-0.26(-0.49,-0.02) & 14.5 \\
-0.13(-0.45,0.19) & 12.3 \\
-0.22(-0.41,-0.03) & 15.5 \\
-0.32(-0.52,-0.12) & 100.0
\end{array}
$$

ES

Fig. 2 Association between total symptoms and (a) understanding; (b) appreciation; (c) reasoning. ES, effect size.

and if they present with severe psychotic symptoms and poor verbal cognitive functioning. Conversely, people with psychosis are more likely to be judged to retain the capacity to make their own decisions if they are relatively well educated, if they demonstrate a reflective 'metacognitive' awareness of their difficulties, and if they experience less severe psychotic symptoms or cognitive impairment. Although there is preliminary evidence that heightened anxiety may also be associated with a reduced risk of incapacity in psychosis, depression does not at present seem to be an important factor.

Overall, our review has shown there is promising evidence that treatment decision-making capacity may be responsive to intervention. On the other hand, it has been at least 25 years since the first study of capacity in psychosis, and we still lack robust evidence from randomised controlled trials to know how to support it. Indeed, the absence of high-quality evidence on interventions to improve capacity precludes recommendation of one particular approach. However, we believe that basic standards in ethical and clinical practice dictate that clinicians should endeavour to take a collaborative approach when seeking to support or restore the capacity of their patients, that they should take all reasonable steps to seek their patients' assent for any capacity-supporting interventions they attempt, that any decisions are informed by a thorough assessment and understanding of the specific predisposing and maintaining factors involved in maintaining that person's impaired capacity, and that they use 


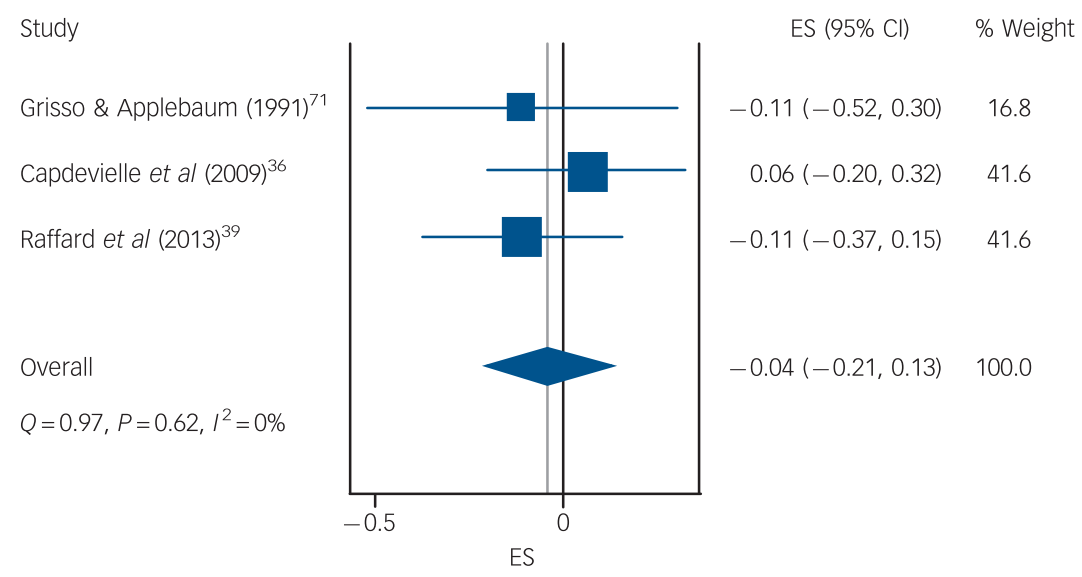

Fig. 3 Association between depression and understanding. ES, effect size.

(a) Study

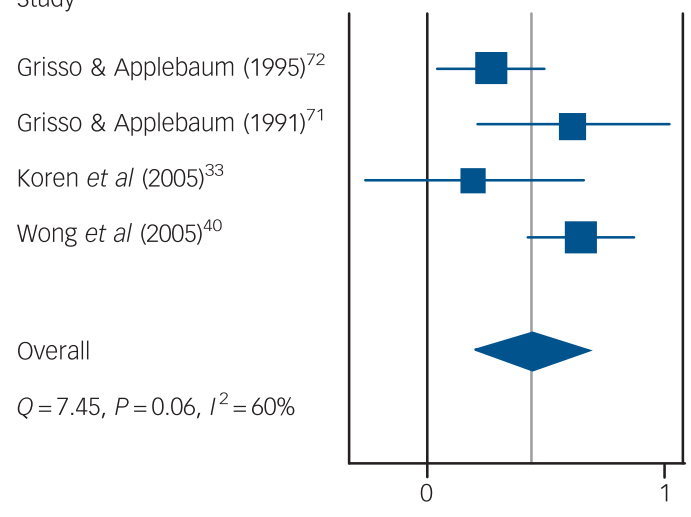

ES

(b) Study

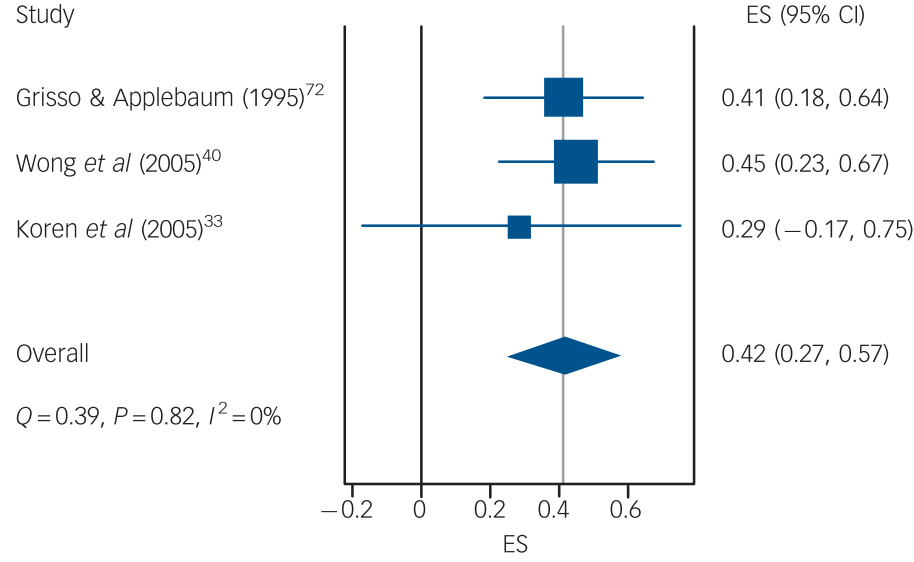

Fig. 4 Association between verbal IQ score and (a) understanding; (b) reasoning. ES, effect size.
ES $(95 \% \mathrm{Cl}) \quad$ \% Weight

$0.27(0.04,0.50) \quad 31.4$

$0.62(0.21,1.03) \quad 19.5$

$0.19(-0.27,0.65) \quad 16.9$

$0.65(0.43,0.87) \quad 32.1$

$0.45(0.20,0.69) \quad 100.0$

$\%$ Weight

42.9

46.4

10.7

100.00 the least invasive (and safest) capacity-supporting interventions available to them. It is likely that interventions meeting this last criterion will include collaborative decision-making and simplification and repetition of decision-relevant information, as well as more complex psychological interventions such as metacognitive training, cognitive remediation and cognitivebehavioural therapy. The latter are relatively 'tried and tested' psychological treatments for psychosis, and we know they have beneficial effects on some of the correlates of impaired capacity we have identified - namely symptoms, ${ }^{50,51}$ metacognition,,${ }^{51-53}$ and cognition. ${ }^{54}$ Nonetheless, the current absence of direct evidence means that clinicians cannot assume such approaches are effective for supporting capacity, or that they are free from adverse effects. ${ }^{45}$
For example, it is entirely plausible that improvements in capacity could be accompanied by increased emotional distress, ${ }^{36,39}$ perhaps because of increased insight, self-stigma or hopelessness. ${ }^{5,56}$ This uncertainty therefore underlines the importance of clinicians carefully evaluating the success, safety and acceptability of their capacity-supporting interventions.

\section{Study limitations}

Some may object to capacity being treated as a continuous variable in the meta-analyses, noting that in legal and clinical practice binary decisions must be made. However, continuous and categorical approaches to classification in psychiatric research 
(a)

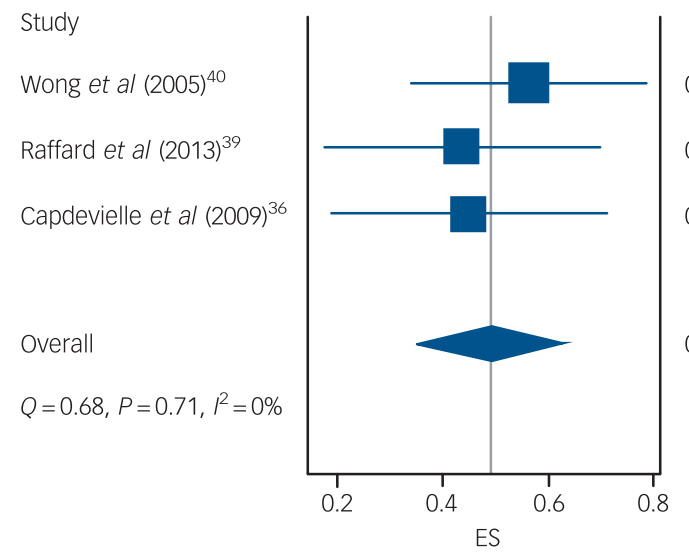

(b)

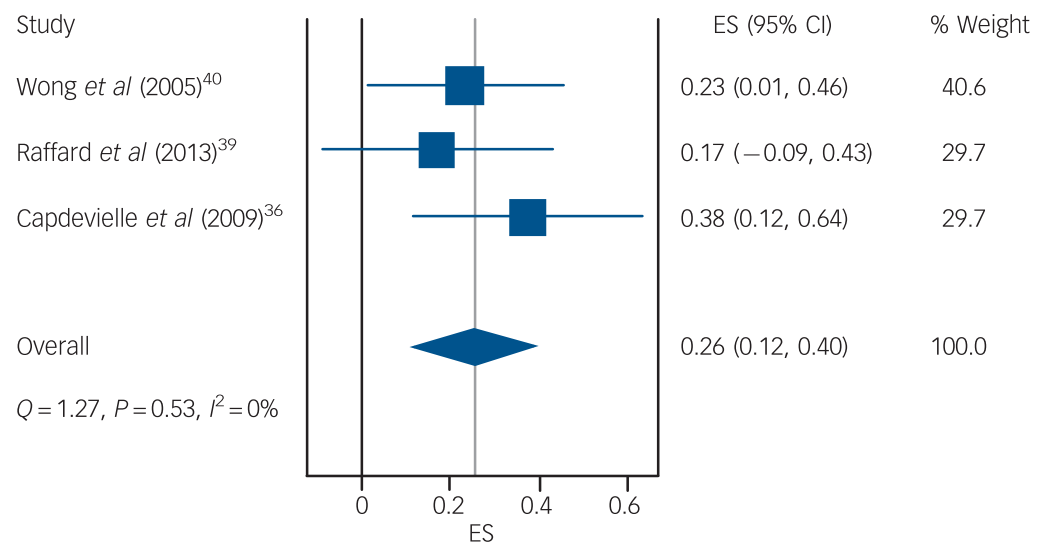

ES $(95 \% \mathrm{Cl}) \quad$ \% Weight

$0.56(0.34,0.78) \quad 40.6$

$0.44(0.18,0.70) \quad 29.7$

$0.45(0.19,0.71) \quad 29.7$

$0.49(0.35,0.63) \quad 100.0$

$\%$ Weight

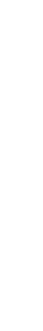

\section{Fig. 5 Association between years of education and (a) understanding; (b) reasoning. ES, effect size.}

and practice are not necessarily mutually exclusive. At this early stage in our understanding of capacity in psychosis, we believe that both approaches can and should be used. Relying only on comparisons between those who have and do not have capacity is problematic for a number of reasons. For example, dichotomising continuous variables is associated with a significant loss of statistical power, equivalent to discarding a third of the data. ${ }^{57}$ Dichotomising also masks the fact that people who have borderline impaired capacity may differ much more from someone with severely impaired capacity than they do from someone with borderline intact capacity. Thus, analysing capacity only as a binary construct may lead to incorrect conclusions about the underlying factors that help or hinder capacity.

We originally decided that studies that did not use assessors masked to clinical status when assessing capacity were lower in quality than those that did. However, we acknowledge that this approach does not recognise that real-life judgements of impaired capacity often require clinicians to decide first that a mental disorder is present, and that capacity assessment often involves assessing a person's views on their diagnosis, something which is clearly incompatible with assessor masking. The fact that assessors need to know a diagnosis to perform a thorough capacity assessment does not negate the possibility that such assessments are subject to bias. Without some degree of masking, there remains a significant risk that assessors' beliefs about particular diagnoses may influence the way in which they appraise the values and beliefs of the people they are assessing.

\section{Implications}

The concept of capacity was developed partly in response to widespread recognition that status-based tests of competence lack validity. However, there is a concern that capacity has become a simple proxy for insight for many clinicians, ${ }^{58}$ thus allowing status-based tests of competence to continue to exert undue influence, albeit in a less obvious way. ${ }^{59}$ If decisional capacity is to be accepted as a valid proxy for patient autonomy, however, then it must take seriously those definitions of recovery and self-governance advocated by patients, as well as the existence of competing explanatory frameworks. ${ }^{60}$ Given that recovery of the ability to self-govern in relation to psychiatric treatment is without doubt an outcome of great importance to many service users with psychosis, further research and analysis in this area is required.

The correlational nature of much of the data in this metaanalysis limits a definitive assessment of causality. Experimental studies conducted within a causal-interventionist framework are now required to develop and test a theoretical model of capacity in psychosis. ${ }^{61}$ It is also important to consider the wealth of research on cognitive and neuropsychological factors involved in less emotionally salient or 'real-life' decision-making - for example, as measured by the Iowa Gambling Task. The development of a comprehensive theory of capacity in psychosis will require integration and synthesis of this literature, but this was outwith the scope of this review.

\section{Future research}

Although some researchers have started to adapt and apply more sophisticated non-pharmacological therapeutic approaches to impaired capacity, ${ }^{15}$ we still lack a good model to inform treatment development. Future research might usefully examine the role of reasoning biases, ${ }^{62,63}$ attitudes and beliefs, ${ }^{64}$ emotions such as fear or anxiety, ${ }^{65}$ and values. ${ }^{66}$ The findings of such studies 
could have important implications for current concepts of decisional capacity in psychosis, and how these interact with the underlying models held by those carrying out capacity assessments - be they primarily social, ${ }^{67}$ psychological, ${ }^{62,68}$ or biological. ${ }^{69}$

Amanda Larkin, DClinPsych, Psychosis Research Unit, Greater Manchester Menta Health National Health Service Foundation Trust, Prestwich; Paul Hutton, DClinPsych, School of Health and Social Care, Edinburgh Napier University, Edinburgh, UK

Correspondence: Amanda Larkin, Psychosis Research Unit, Rico House, Prestwich M25 3BL, UK. Email: amanda.larkin@nhs.net

First received 16 Sep 2016, final revision 4 Feb 2017, accepted 14 Feb 2017

\section{Acknowledgements}

We thank all those authors who provided additional information about their studies. We would also like to thank anonymous reviewers for their high-quality reviews and helpfu comments and suggestions.

\section{References}

1 Appelbaum PS, Grisso T. The MacArthur Treatment Competence Study. I: Mental illness and competence to consent to treatment. Law Hum Behav 1995; 19: 105-26.

2 Owen GS, Freyenhagen F, Richardson G, Hotopf M. Mental capacity and decisional autonomy: an interdisciplinary challenge. Inquiry 2009; $\mathbf{5 1}$ 79-107.

3 Pitt L, Kilbride M, Nothard S, Welford M, Morrison A. Researching recovery from psychosis: a user-led project. Psychiatr Bull 2007; 31: 55-60.

4 Law H, Morrison AP. Recovery in psychosis: a Delphi study with experts by experience. Schizophr Bull 2014; 40: 1347-55.

5 Lepping P, Raveesh BN. Overvaluing autonomous decision-making. $\mathrm{Br} \mathrm{J}$ Psychiatry 2014; 204: 1-2.

6 Donnelly MMA. Healthcare Decision-Making and The Law: Autonomy, Capacity and the Limits of Liberalism. Cambridge University Press, 2010.

7 Lepping $\mathrm{P}$, Stanly $\mathrm{T}$, Turner J. Systematic review on the prevalence of lack of capacity in medical and psychiatric settings. Clin Med (Lond) 2015; 15 337-43

8 Seyfried L, Ryan KA, Kim SY. Assessment of decision-making capacity: views and experiences of consultation psychiatrists. Psychosomatics 2013; 54: $115-23$

9 Hamann J, Cohen R, Leucht S, Busch R, Kissling W. Do patients with schizophrenia wish to be involved in decisions about their medical treatment? Am J Psychiatry 2005; 162: 2382-4.

10 National Collaborating Centre for Mental Health. Psychosis and Schizophrenia in Adults: Prevention and Management (Clinical Guideline CG178). NICE, 2014.

11 Royal College of Psychiatrists. Report of the Second Round of the National Audit of Schizophrenia (NAS) 2014. Healthcare Quality Improvement Partnership, 2014.

12 Hamann J, Mendel R, Cohen R, Heres S, Ziegler M, Buhner M, et al. Psychiatrists' use of shared decision making in the treatment of schizophrenia: patient characteristics and decision topics. Psychiatr Serv 2009; 60: 1107-12.

13 British Medical Association, Law Society. Assessment of Mental Capacity: A Practical Guide for Doctors and Lawyers (4th edn). Law Society Publishing, 2015

14 Carpenter WT, Gold JM, Lahti AC, Queern CA, Conley RR, Bartko JJ, et al. Decisional capacity for informed consent in schizophrenia research. Arch Gen Psychiatry 2000; 57: 533-8.

15 Naughton M, Nulty A, Abidin Z, Davoren M, O'Dwyer S, Kennedy HG. Effects of group metacognitive training (MCT) on mental capacity and functioning in patients with psychosis in a secure forensic psychiatric hospital: a prospective-cohort waiting list controlled study. BMC Res Notes 2012; 5: 302.

16 Okai D, Owen G, McGuire H, Singh S, Churchill R, Hotopf M. Mental capacity in psychiatric patients: systematic review. Br J Psychiatry 2007; 191: 291-7.

17 Dunn LB. Capacity to consent to research in schizophrenia: the expanding evidence base. Behav Sci Law 2006; 24: 431-45.

18 Sturman ED. The capacity to consent to treatment and research: a review of standardized assessment tools. Clin Psychol Rev 2005; 25: 954-74.

19 Jeste DV, Depp CA, Palmer BW. Magnitude of impairment in decisional capacity in people with schizophrenia compared to normal subjects: an overview. Schizophr Bull 2006; 32: 121-8.
20 Ruissen AM, Widdershoven GA, Meynen G, Abma TA, van Balkom AJ. A systematic review of the literature about competence and poor insight. Acta Psychiatr Scand 2012; 125: 103-13.

21 Palmer BW, Savla GN. The association of specific neuropsychological deficits with capacity to consent to research or treatment. J Int Neuropsychol SoC 2007; 13: 1047-59.

22 Guyatt GH, Oxman AD, Vist GE, Kunz R, Falck-Ytter $Y$, Alonso-Coello P, et al. GRADE: an emerging consensus on rating quality of evidence and strength of recommendations. BMJ 2008; 336: 924-6.

23 Taylor PJ, Hutton P, Wood L. Are people at risk of psychosis also at risk of suicide and self-harm? A systematic review and meta-analysis. Psychol Med 2015; 45: 911-26.

24 Dudley R, Taylor P, Wickham S, Hutton P. Psychosis, delusions and the 'jumping to conclusions' reasoning bias: a systematic review and metaanalysis. Schizophr Bull 2016; 42: 652-65.

25 Williams JW, Plassman BL, Burke J, Holsinger T, Benjamin S. Preventing Alzheimer's Disease and Cognitive Decline. Evidence Report/Technology Assessment 193. US Agency for Healthcare Research and Quality, 2010.

26 Higgins JP, Altman DG, Gotzsche PC, Juni P, Moher D, Oxman AD, et al. The Cochrane Collaboration's tool for assessing risk of bias in randomised trials. BMJ 2011; 343: d5928.

27 DerSimonian R, Laird N. Meta-analysis in clinical trials. Control Clin Trials 1986; 7: 177-88

28 Borenstein M. Introduction to Meta-Analysis. Wiley, 2009.

29 Cohen J. Statistical Power Analysis For The Behavioral Sciences (2nd edn). Erlbaum, 1988.

30 Ioannidis JP, Trikalinos TA. The appropriateness of asymmetry tests for publication bias in meta-analyses: a large survey. CMAJ 2007; 176: 1091-6.

31 Grisso T, Appelbaum PS, Hill-Fotouhi C. The MacCAT-T: a clinical tool to assess patients' capacities to make treatment decisions. Psychiatr Serv 1997; 48: 1415-9.

32 Rutledge E, Kennedy M, O'Neill H, Kennedy HG. Functional mental capacity is not independent of the severity of psychosis. Int J Law Psychiatry 2008; 31: 9-18.

33 Koren D, Poyurovsky M, Seidman $\sqcup$, Goldsmith $M$, Wenger S, Klein EM. The neuropsychological basis of competence to consent in first-episode schizophrenia: a pilot metacognitive study. Biol Psychiatry 2005; 57: 609-16.

34 Mandarelli G, Parmigiani G, Tarsitani L, Frati P, Biondi M, Ferracuti S. The relationship between executive functions and capacity to consent to treatment in acute psychiatric hospitalization. J Empir Res Hum Res Ethics 2012; 7: 63-70.

35 Cairns R, Maddock C, Buchanan A, David AS, Hayward P, Richardson G, et al. Reliability of mental capacity assessments in psychiatric in-patients. Br J Psychiatry 2005; 187: 372-8.

36 Capdevielle D, Raffard S, Bayard S, Garcia F, Baciu O, Bouzigues I, et al. Competence to consent and insight in schizophrenia: is there an association? A pilot study. Schizophr Res 2009; 108: 272-9.

37 Owen GS, David AS, Richardson G, Szmukler G, Hayward P, Hotopf M. Mental capacity, diagnosis and insight in psychiatric in-patients: a cross-sectional study. Psychol Med 2009; 39: 1389-98.

38 Elbogen EB, Swanson JW, Appelbaum PS, Swartz MS, Ferron J, Van Dorn RA, et al. Competence to complete psychiatric advance directives: effects of facilitated decision making. Law Hum Behav 2007; 31: 275-89.

39 Raffard S, Fond G, Brittner M, Bortolon C, Macgregor A, Boulenger JP, et al. Cognitive insight as an indicator of competence to consent to treatment in schizophrenia. Schizophr Res 2013; 144: 118-21.

40 Wong JG, Cheung EP, Chen EY. Decision-making capacity of inpatients with schizophrenia in Hong Kong. J Nerv Ment Dis 2005; 193: 316-22.

41 Moritz S, Woodward TS, Balzan R. Is metacognitive training for psychosis effective? Expert Rev Neurother 2016; 16: 105-7.

42 Munetz MR, Roth LH. Informing patients about tardive dyskinesia. Arch Gen Psychiatry 1985; 42: 866-71.

43 Palmer BW, Nayak GV, Dunn LB, Appelbaum PS, Jeste DV. Treatment-related decision-making capacity in middle-aged and older patients with psychosis: a preliminary study using the MacCAT-T and HCAT. Am J Geriatr Psychiatry 2002; 10: 207-11.

44 Kleinman I, Schachter D, Jeffries J, Goldhamer P. Informed consent and tardive dyskinesia. Long-term follow-up. J Nerv Ment Dis 1996; 184: 517-22.

45 Kennedy M, Dornan J, Rutledge E, O'Neill H, Kennedy HG. Extra information about treatment is too much for the patient with psychosis. Int J Law Psychiatry 2009; 32: 369-76.

46 Wong JG, Clare $\mathrm{CH}$, Holland AJ, Watson PC, Gunn M. The capacity of people with a 'mental disability' to make a health care decision. Psychol Med 2000; 30: 295-306. 
47 Owen GS, Ster IC, David AS, Szmukler G, Hayward P, Richardson G, et al. Regaining mental capacity for treatment decisions following psychiatric admission: a clinico-ethical study. Psychol Med 2011; 41: 119-28.

48 Dornan J, Kennedy M, Garland J, Rutledge E, Kennedy HG. Functional menta capacity, treatment as usual and time: magnitude of change in secure hospital patients with major mental illness. BMC Res Notes 2015; 8: 566.

49 Hamann J, Mendel R, Meier A, Asani F, Pausch E, Leucht S, et al. 'How to speak to your psychiatrist': shared decision-making training for inpatients with schizophrenia. Psychiatr Serv 2011; 62: 1218-21.

50 Turner DT, van der Gaag M, Karyotaki E, Cuijpers P. Psychological interventions for psychosis: a meta-analysis of comparative outcome studies. Am J Psychiatry 2014; 171: 523-38.

51 Eichner C, Berna F. Acceptance and efficacy of metacognitive training (MCT) on positive symptoms and delusions in patients with schizophrenia: a meta-analysis taking into account important moderators. Schizophr Bull 2016; 42: 952-62.

52 Cella M, Preti A, Edwards C, Dow T, Wykes T. Cognitive remediation for negative symptoms of schizophrenia: a network meta-analysis. Clin Psycho Rev 2016; 52: 43-51.

53 Cella $\mathrm{M}$, Reeder $\mathrm{C}$, Wykes T. Lessons learnt? The importance of metacognition and its implications for cognitive remediation in schizophrenia. Front Psychol 2015; 6: 1259.

54 Wykes T, Huddy V, Cellard C, McGurk SR, Czobor P. A meta-analysis of cognitive remediation for schizophrenia: methodology and effect sizes. Am J Psychiatry 2011; 168: 472-85.

55 Belvederi Murri $M$, Respino $M$, Innamorati $M$, Cervetti $A$, Calcagno $P$, Pompili $M$, et al. Is good insight associated with depression among patients with schizophrenia? Systematic review and meta-analysis. Schizophr Res 2015; 162: 234-47.

56 Belvederi Murri $\mathrm{M}$, Amore $\mathrm{M}$, Calcagno $\mathrm{P}$, Respino $\mathrm{M}$, Marozzi V, Masotti $\mathrm{M}$ et al. The 'insight paradox' in schizophrenia: magnitude, moderators and mediators of the association between insight and depression. Schizophr Bull 2016; 42: 1225-33.

57 Altman DG, Royston P. The cost of dichotomising continuous variables. BMJ 2006; 332: 1080.

58 Shek E, Lyons D, Taylor M. Understanding 'significant impaired decisionmaking ability' with regard to treatment for mental disorder: an empirical analysis. The Psychiatrist 2010; 34: 239-42.

59 Allen N. Is capacity "in sight"? J Ment Health Law 2009; Winter: 165-70.

60 Cooke A (ed). Understanding Psychosis and Schizophrenia: Why People Sometimes Hear Voices, Believe Things that Others Find Strange, or Appear
Out of Touch with Reality and what Can Help. British Psychological Society, 2014

61 Kendler KS, Campbell J. Interventionist causal models in psychiatry: repositioning the mind-body problem. Psychol Med 2009; 39: 881-7.

62 Garety PA, Kuipers E, Fowler D, Freeman D, Bebbington PE. A cognitive model of the positive symptoms of psychosis. Psychol Med 2001; 31: 189-95.

63 Kahneman D. A perspective on judgment and choice: mapping bounded rationality. Am Psychol 2003; 58: 697-720.

64 Armitage $\mathrm{CJ}$, Conner M. Efficacy of the theory of planned behaviour: a metaanalytic review. Br J SOC Psychol 2001; 40(Pt 4): 471-99.

65 Hartley CA, Phelps EA. Anxiety and decision-making. Biol Psychiatry 2012; 72: $113-8$.

66 Mukherjee D, Kable JW. Value-based decision-making in mental illness: a meta-analysis. Clin Psychol Sci 2014; 2: 767-82.

67 Selten JP, van der Ven E, Rutten BP, Cantor-Graae E. The social defeat hypothesis of schizophrenia: an update. Schizophr Bull 2013; 39: 1180-6.

68 Morrison AP. The interpretation of intrusions in psychosis: an integrative cognitive approach to hallucinations and delusions. Behav Cogn Psychother 2001; 29: 257-76.

69 Howes OD, Murray RM. Schizophrenia: an integrated sociodevelopmentalcognitive model. Lancet 2014; 383: 1677-87.

$70 \mathrm{Di} \mathrm{X}$, Cheng $\mathrm{H}$. Competence of consent and associated factors among inpatients of schizophrenia in Changsa, China. J Schizophr Res 2013; 150: 325-6.

71 Grisso T, Appelbaum PS. Mentally ill and non-mentally-ill patients' abilities to understand informed consent disclosures for medication: preliminary data. Law Hum Behav 1991; 15: 377.

72 Grisso T, Appelbaum PS. The MacArthur Treatment Competence Study. III: Abilities of patients to consent to psychiatric and medical treatments. Law Hum Behav 1995; 19: 149

73 Howe V, Foister K, Jenkins K, Stene L, Copolov D, Neks N. Competence to give informed consent in acute psychosis is associated with symptoms rather than diagnosis. Schizophr Res 2005; 77: 211-4.

74 Owen GS, Richardson G, David AS, Szmukler G, Hayward P, Hotopf M. Mental capacity to make decisions on treatment in people admitted to psychiatric hospitals: cross sectional study. BMJ 2008; 337: a448.

75 Schacter D, Kleinman I, Prendergast P, Remington G, Schertzer S. The effect of psychopathology on the ability of schizophrenic patients to give informed consent. J Nerv Ment Dis 1994; 182: 360-2. 